\title{
Hodge Theory of Matroids
}

Editor's Note: Matt Baker is speaking on this topic in the Current Events Bulletin Lecture at the January 2017 Joint Mathematics Meetings.

\section{Karim Adiprasito, June Huh, and Eric Katz}

\author{
Communicated by Benjamin Braun
}

\section{Introduction}

Logarithmic concavity is a property of a sequence of real numbers, occurring throughout algebraic geometry, convex geometry, and combinatorics. A sequence of positive numbers $a_{0}, \ldots, a_{d}$ is log-concave if

$$
a_{i}^{2} \geq a_{i-1} a_{i+1} \text { for all } i \text {. }
$$

This means that the $\operatorname{logarithms}, \log \left(a_{i}\right)$, form a concave sequence. The condition implies unimodality of the sequence $\left(a_{i}\right)$, a property easier to visualize: the sequence is unimodal if there is an index $i$ such that

$$
a_{0} \leq \cdots \leq a_{i-1} \leq a_{i} \geq a_{i+1} \geq \cdots \geq a_{d} .
$$

We will discuss our work on establishing log-concavity of various combinatorial sequences, such as the coefficients of the chromatic polynomial of graphs and the face numbers of matroid complexes. Our method is motivated by complex algebraic geometry, in particular Hodge theory. From a given combinatorial object $M$ (a matroid), we construct a graded commutative algebra over the real numbers

$$
A^{*}(M)=\bigoplus_{q=0}^{d} A^{q}(M),
$$

Karim Adiprasito is assistant professor of mathematics at Hebrew University of Jerusalem. His e-mail address is adiprasi to@math. huji.ac.i1.

June Huh is research fellow at the Clay Mathematics Institute and Veblen Fellow at Princeton University and the Institute for Advanced Study. His e-mail is huh@princeton .edu.

Eric Katz is assistant professor of mathematics at the Ohio State University. His e-mail address is katz.60@osu. edu.

For permission to reprint this article, please contact: reprint-permission@ams.org.

DOI: http://dx.doi.org/10.1090/noti1463 which satisfies analogues of Poincaré duality, the hard Lefschetz theorem, and the Hodge-Riemann relations for the cohomology of smooth projective varieties. Log-concavity will be deduced from the Hodge-Riemann relations for $M$. We believe that behind any log-concave sequence that appears in nature there is such a "Hodge structure" responsible for the log-concavity.

\section{Coloring Graphs}

Generalizing earlier work of George Birkhoff, in 1932 Hassler Whitney introduced the chromatic polynomial of a connected graph $G$ as the function on $\mathbb{N}$ defined by

$$
\chi_{G}(q)=\mid\{\text { proper colorings of } G \text { using } q \text { colors }\} \mid \text {. }
$$

In other words, $\chi_{G}(q)$ is the number of ways to color the vertices of $G$ using $q$ colors so that the endpoints of every edge have different colors. Whitney noticed that the chromatic polynomial is indeed a polynomial. In fact, we can write

$$
\chi_{G}(q) / q=a_{0}(G) q^{d}-a_{1}(G) q^{d-1}+\cdots+(-1)^{d} a_{d}(G)
$$

for some positive integers $a_{0}(G), \ldots, a_{d}(G)$, where $d$ is one less than the number of vertices of $G$.

Example 1. The square graph

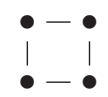

has the chromatic polynomial $1 q^{4}-4 q^{3}+6 q^{2}-3 q$.

The chromatic polynomial was originally devised as a tool for attacking the Four Color Problem, but soon it attracted attention in its own right. Ronald Read conjectured in 1968 that the coefficients of the chromatic polynomial form a unimodal sequence for any graph. 
A few years later, Stuart Hoggar conjectured that the coefficients in fact form a log-concave sequence:

$$
a_{i}(G)^{2} \geq a_{i-1}(G) a_{i+1}(G) \text { for any } i \text { and } G .
$$

The graph theorist William Tutte quipped, "In compensation for its failure to settle the Four Colour Conjecture, [the chromatic polynomial] offers us the Unimodal Conjecture for our further bafflement."

The chromatic polynomial can be computed using the deletion-contraction relation: if $G \backslash e$ is the deletion of an edge $e$ from $G$ and $G / e$ is the contraction of the same edge, then

$$
\chi_{G}(q)=\chi_{G \backslash e}(q)-\chi_{G / e}(q) .
$$

The first term counts the proper colorings of $G$, the second term counts the otherwise-proper colorings of $G$ where the endpoints of $e$ are permitted to have the same color, and the third term counts the otherwise-proper colorings of $G$ where the endpoints of $e$ are mandated to have the same color. Note that, in general, the sum of two $\log$-concave sequences is not a log-concave sequence.

Example 2. To compute the chromatic polynomial of the square graph above, we write

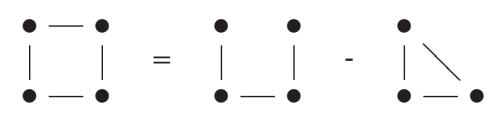

and use

$$
\chi_{G \backslash e}(q)=q(q-1)^{3}, \quad \chi_{G / e}(q)=q(q-1)(q-2) .
$$

The Hodge-Riemann relations for the algebra $A^{*}(M)$, where $M$ is the matroid attached to $G$ as in the section "Matroids" below, imply that the coefficients of the chromatic polynomial of $G$ form a log-concave sequence. This is in contrast to a result of Alan Sokal that the set of roots of the chromatic polynomials of all graphs is dense in the complex plane.

\section{Counting Independent Subsets}

Linear independence is a fundamental notion in algebra and geometry: a collection of vectors is linearly independent if no nontrivial linear combination sums to zero. How many linearly independent collections of $i$ vectors are there in a given configuration of vectors? Write $A$ for a finite subset of a vector space and $f_{i}(A)$ for the number of independent subsets of $A$ of size $i$.

Example 3. If $A$ is the set of all nonzero vectors in the three-dimensional vector space over the field with two elements, then

$$
f_{0}=1, \quad f_{1}=7, f_{2}=21, \quad f_{3}=28 .
$$

Examples suggest a pattern leading to a conjecture of Dominic Welsh:

$$
f_{i}(A)^{2} \geq f_{i-1}(A) f_{i+1}(A) \text { for any } i \text { and } A .
$$

For any small specific case, the conjecture can be verified by computing the $f_{i}(A)$ 's by the deletion-contraction relation: if $A \backslash v$ is the deletion of a nonzero vector $v$ from $A$ and $A / v$ is the projection of $A$ in the direction of $v$, then

$$
f_{i}(A)=f_{i}(A \backslash \nu)+f_{i-1}(A / v) \text {. }
$$

The first term counts the number of independent subsets of size $i$, the second term counts the independent subsets of size $i$ not containing $v$, and the third term counts the independent subsets of size $i$ containing $v$. As in the case of graphs, we notice the apparent interference between the log-concavity conjecture and the additive nature of $f_{i}(A)$. The sum of two log-concave sequences is, in general, not log-concave. The conjecture suggests a new description for the numbers $f_{i}(A)$ and a corresponding structure of $A$.

\section{Matroids}

In the 1930s Hassler Whitney observed that several notions in graph theory and linear algebra fit together in a common framework, that of matroids. This observation started a new subject with applications to a wide range of topics such as characteristic classes, optimization, and moduli spaces, to name a few.

Let $E$ be a finite set. A matroid $M$ on $E$ is a collection of subsets of $E$, called flats of $M$, satisfying the following axioms:

(1) If $F_{1}$ and $F_{2}$ are flats of $M$, then their intersection is a flat of $M$.

(2) If $F$ is a flat of $M$, then any element of $E \backslash F$ is contained in exactly one flat of $M$ covering $F$.

(3) The ground set $E$ is a flat of $M$.

Here, a flat of $M$ is said to cover $F$ if it is minimal among the flats of $M$ properly containing $F$. For our purposes, we may assume that $M$ is loopless:

(1) The empty subset of $E$ is a flat of $M$.

Every maximal chain of flats of $M$ has the same length, and this common length is called the rank of $M$. We write $M \backslash e$ for the matroid obtained by deleting $e$ from the flats of $M$ and $M / e$ for the matroid obtained by deleting $e$ from the flats of $M$ containing $e$. When $M_{1}$ is a matroid on $E_{1}$, $M_{2}$ is a matroid on $E_{2}$, and $E_{1} \cap E_{2}$ is empty, the direct sum $M_{1} \oplus M_{2}$ is defined to be the matroid on $E_{1} \cup E_{2}$ whose Matroids encode a combinatorial structure common to graphs and vector configurations

form $F_{1} \cup F_{2}$, where $F_{1}$ is a flat of $M_{1}$ and $F_{2}$ is a flat of $M_{2}$.

Matroids encode a combinatorial structure common to graphs and vector configurations. If $E$ is the set of edges of a finite graph $G$, call a subset $F$ of $E$ a flat when there is no edge in $E \backslash F$ whose endpoints are connected by a path in $F$. This defines a graphic matroid on $E$. If $E$ is a finite subset of a vector space, call a subset $F$ of $E$ a flat when there is no vector in $E \backslash F$ that is contained in the linear span of $F$. This defines a linear matroid on $E$. 
Example 4. Write $E=\{0,1,2,3\}$ for the set of edges of the square graph $G$ in Example 1. The graphic matroid $M$ on $E$ attached to $G$ has flats

$$
\begin{aligned}
\varnothing,\{0\},\{1\},\{2\},\{3\},\{0,1\},\{0,2\},\{0,3\}, \\
\{1,2\},\{1,3\},\{2,3\},\{0,1,2,3\} .
\end{aligned}
$$

Example 5. Write $E=\{0,1,2,3,4,5,6\}$ for the configuration of vectors $A$ in Example 3. The linear matroid $M$ on $E$ attached to $A$ has flats

$$
\begin{array}{r}
\varnothing,\{0\},\{1\},\{2\},\{3\},\{4\},\{5\},\{6\}, \\
\{1,2,3\},\{3,4,5\},\{1,5,6\},\{0,1,4\},\{0,2,5\},\{0,3,6\}, \\
\{2,4,6\},\{0,1,2,3,4,5,6\} .
\end{array}
$$

A linear matroid that arises from a subset of a vector space over a field $k$ is said to be realizable over $k$. Not surprisingly, this notion is sensitive to the field $k$. A matroid may arise from a vector configuration over one field, while no such vector configuration exists over another field. Many matroids are not realizable over any field.
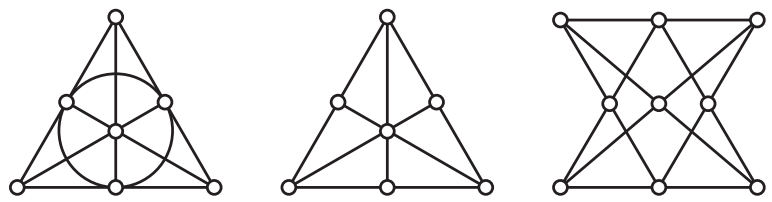

Among the rank 3 loopless matroids pictured above, where rank 1 flats are represented by points and rank 2 flats containing more than two points are represented by lines, the first is realizable over $k$ if and only if the characteristic of $k$ is 2 , the second is realizable over $k$ if and only if the characteristic of $k$ is not 2, and the third is not realizable over any field.

The characteristic polynomial $\chi_{M}(q)$ of a matroid $M$ is a generalization of the chromatic polynomial $\chi_{G}(q)$ of a graph $G$. It can be recursively defined using the following rules:

(1) If $M$ is the direct sum $M_{1} \oplus M_{2}$, then

$$
\chi_{M}(q)=\chi_{M_{1}}(q) \chi_{M_{2}}(q) .
$$

(2) If $M$ is not a direct sum, then, for any $e$,

$$
\chi_{M}(q)=\chi_{M \backslash e}(q)-\chi_{M / e}(q) .
$$

(3) If $M$ is the rank 1 matroid on $\{e\}$, then

$$
\chi_{M}(q)=q-1 .
$$

(4) If $M$ is the rank 0 matroid on $\{e\}$, then

$$
\chi_{M}(q)=0 .
$$

It is a consequence of the Möbius inversion for partially ordered sets that the characteristic polynomial of $M$ is well defined.

We may now state our result in [AHK], which confirms a conjecture of Gian-Carlo Rota and Dominic Welsh.

Theorem 6 ([AHK, Theorem 9.9]). The coefficients of the characteristic polynomial form a log-concave sequence for any matroid $M$.

This implies the log-concavity of the sequence $a_{i}(G)$ [Huh12] and the log-concavity of the sequence $f_{i}(A)$ [Len12].
Hodge-Riemann Relations for Matroids

Let $X$ be a mathematical object of "dimension" $d$. Often it is possible to construct from $X$ in a natural way a graded vector space over the real numbers

$$
A^{*}(X)=\bigoplus_{q=0}^{d} A^{q}(X),
$$

equipped with a graded bilinear pairing

$$
P: A^{*}(X) \times A^{d-*}(X) \longrightarrow \mathbb{R},
$$

and a graded linear map

$$
L: A^{*}(X) \longrightarrow A^{*+1}(X), \quad x \longmapsto \mathrm{Lx}
$$

(" $P$ " is for Poincaré, and " $L$ " is for Lefschetz). For example, $A^{*}(X)$ may be the cohomology of real $(p, p)$-forms on a compact Kähler manifold $X$ or the ring of algebraic cycles modulo homological equivalence on a smooth projective variety $X$ or the combinatorial intersection cohomology of a convex polytope $X$ [Kar04] or the Soergel bimodule of an element of a Coxeter group $X$ [EW14] or the Chow ring of a matroid $X$ defined below. We expect that, for every nonnegative integer $q \leq \frac{d}{2}$ :

(1) the bilinear pairing

$$
P: A^{q}(X) \times A^{d-q}(X) \longrightarrow \mathbb{R}
$$

is nondegenerate (Poincaré duality for $X$ ),

(2) the composition of linear maps

$$
L^{d-2 q}: A^{q}(X) \longrightarrow A^{d-q}(X)
$$

is bijective (the hard Lefschetz theorem for $X$ ), and

(3) the bilinear form on $A^{q}(X)$ defined by

$$
\left(x_{1}, x_{2}\right) \longmapsto(-1)^{q} P\left(x_{1}, L^{d-2 q} X_{2}\right)
$$

is symmetric and is positive definite on the kernel of

$$
L^{d-2 q+1}: A^{q}(X) \longrightarrow A^{d-q+1}(X)
$$

(the Hodge-Riemann relations for $X$ ).

All three properties are known to hold for the objects listed above except one, which is the subject of Grothendieck's standard conjectures on algebraic cycles.

For a loopless matroid $M$ on $E$, the vector space $A^{*}(M)$ has the structure of a graded algebra that can be described explicitly.

Definition 7. We introduce variables $x_{F}$, one for each nonempty proper flat $F$ of $M$, and set

$$
S^{*}(M)=\mathbb{R}\left[x_{F}\right]_{F \neq \varnothing, F \neq E} .
$$

The Chow ring $A^{*}(M)$ of $M$ is the quotient of $S^{*}(M)$ by the ideal generated by the linear forms

$$
\sum_{i_{1} \in F} x_{F}-\sum_{i_{2} \in F} x_{F},
$$

one for each pair of distinct elements $i_{1}$ and $i_{2}$ of $E$, and the quadratic monomials

$$
x_{F_{1}} x_{F_{2}},
$$

one for each pair of incomparable nonempty proper flats $F_{1}$ and $F_{2}$ of $M$. 
The Chow ring of $M$ was introduced by Eva Maria Feichtner and Sergey Yuzvinsky. When $M$ is realizable over a field $k$, it is the Chow ring of the "wonderful" compactification of the complement of a hyperplane arrangement defined over $k$ as described by Corrado de Concini and Claudio Procesi.

Let $d$ be the integer one less than the rank of $M$.

Theorem 8 ([AHK, Proposition 5.10]). There is a linear bijection

$$
\operatorname{deg}: A^{d}(M) \rightarrow \mathbb{R}
$$

uniquely determined by the property that

$$
\operatorname{deg}\left(x_{F_{1}} x_{F_{2}} \cdots x_{F_{d}}\right)=1
$$

for every maximal chain of nonempty proper flats

$$
F_{1} \subsetneq F_{2} \subsetneq \cdots \subsetneq F_{d} \text {. }
$$

In addition, the bilinear pairing

$$
P: A^{q}(M) \times A^{d-q}(M) \rightarrow \mathbb{R}, \quad(x, y) \mapsto \operatorname{deg}(x y)
$$

is nondegenerate for every nonnegative $q \leq d$.

What should be the linear operator $L$ for $M$ ? We collect all valid choices of $L$ in a nonempty open convex cone. The cone is an analogue of the Kähler cone in complex geometry.

Definition 9. A real-valued function $c$ on $2^{E}$ is said to be strictly submodular if

$$
c_{\varnothing}=0, \quad c_{E}=0,
$$

and, for any two incomparable subsets $I_{1}, I_{2} \subseteq E$,

$$
c_{I_{1}}+c_{I_{2}}>c_{I_{1} \cap I_{2}}+c_{I_{1} \cup I_{2}} .
$$

A strictly submodular function $c$ defines an element

$$
L(c)=\sum_{F} c_{F} x_{F} \in A^{1}(M)
$$

that acts as a linear operator by multiplication

$$
A^{*}(M) \longrightarrow A^{*+1}(M), \quad x \longmapsto L(c) x .
$$

The set of all such elements is a convex cone in $A^{1}(M)$.

The main result of [AHK] states that the triple $\left(A^{*}(M), P, L(c)\right)$ satisfies the hard Lefschetz theorem and the Hodge-Riemann relations for every strictly submodular function $c$ :

Theorem 10. Let $q$ be a nonnegative integer less than $\frac{d}{2}$.

(1) The multiplication by $L(c)$ defines an isomorphism

$$
A^{q}(M) \longrightarrow A^{d-q}(M), \quad x \longmapsto L(c)^{d-2 q} x .
$$

(2) The symmetric bilinear form on $A^{q}(M)$ defined by

$$
\left(x_{1}, x_{2}\right) \longmapsto(-1)^{q} P\left(x_{1}, L(c)^{d-2 q} x_{2}\right)
$$

is positive definite on the kernel of $L(c)^{d-2 q+1}$.

The known proofs of the hard Lefschetz theorem and the Hodge-Riemann relations for the different types of objects listed above have certain structural similarities, but there is no known way of deducing one from the others.

\section{Sketch of Proof of Log-Concavity}

We now explain why the Hodge-Riemann relations for $M$ imply log-concavity for $\chi_{M}(q)$. The Hodge-Riemann relations for $M$, in fact, imply that the sequence $\left(m_{i}\right)$ in the expression

$$
\chi_{M}(q) /(q-1)=m_{0} q^{d}-m_{1} q^{d-1}+\cdots+(-1)^{d} m_{d}
$$

is log-concave, which is stronger.

We define two elements of $A^{1}(M)$ : for any $j \in E$, set

$$
\alpha_{M}=\sum_{j \in F} x_{F}, \quad \beta_{M}=\sum_{j \notin F} x_{F} .
$$

The two elements do not depend on the choice of $j$, and they are limits of elements of the form $L(c)$ for strictly submodular $c$. A short combinatorial argument shows that $m_{i}$ is a mixed degree of $\alpha_{M}$ and $\beta_{M}$ :

$$
m_{i}=\operatorname{deg}\left(\alpha_{M}^{i} \beta_{M}^{d-i}\right) .
$$

Thus, it is enough to prove for every $i$ that

$$
\operatorname{deg}\left(\alpha_{M}^{d-i+1} \beta_{M}^{i-1}\right) \operatorname{deg}\left(\alpha_{M}^{d-i-1} \beta_{M}^{i+1}\right) \leq \operatorname{deg}\left(\alpha_{M}^{d-i} \beta_{M}^{i}\right)^{2} .
$$

This is an analogue of the Teisser-Khovanskii inequality for intersection numbers in algebraic geometry and the Alexandrov-Fenchel inequality for mixed volumes in convex geometry. The main case is when $i=d-1$.

By a continuity argument, we may replace $\beta_{M}$ by $L=L(c)$ sufficiently close to $\beta_{M}$. The desired inequality in the main case then becomes

$$
\operatorname{deg}\left(\alpha_{M}^{2} L^{d-2}\right) \operatorname{deg}\left(L^{d}\right) \leq \operatorname{deg}\left(\alpha_{M} L^{d-1}\right)^{2} .
$$

This follows from the fact that the signature of the bilinear form

$$
A^{1}(M) \times A^{1}(M) \rightarrow \mathbb{R}, \quad\left(x_{1}, x_{2}\right) \mapsto \operatorname{deg}\left(x_{1} L^{d-2} x_{2}\right)
$$

restricted to the span of $\alpha_{M}$ and $L$ is semi-indefinite, which, in turn, is a consequence of the Hodge-Riemann relations for $M$ in the cases $q=0,1$.

This application uses only a small piece of the HodgeRiemann relations for $M$. The general Hodge-Riemann relations for $M$ may be used to extract other interesting combinatorial information about $M$.

\section{References}

[AHK] Karim Adiprasito, June HuH, and ERIC KATZ, Hodge theory for combinatorial geometries, arXiv:1511.02888.

[EW14] Elias-Williamson, BeN Elias, and GeORDie Williamson, The Hodge theory of Soergel bimodules, Ann. Math. (2) 180 (2014), 1089-1136. MR3245013

[Huh12] JuNE HuH, Milnor numbers of projective hypersurfaces and the chromatic polynomial of graphs, J. Amer. Math. Soc. 25 (2012), 907-927. MR2904577

[Kar04] KALLE KARU, Hard Lefschetz theorem for nonrational polytopes, Invent. Math. 157 (2004), 419-447. MR2076929

[Len12] MATTHIAS LENZ, The f-vector of a representablematroid complex is log-concave, $A d v$. in Appl. Math. $\mathbf{5 1}$ (2013), no. 5, 543-545. MR3118543 


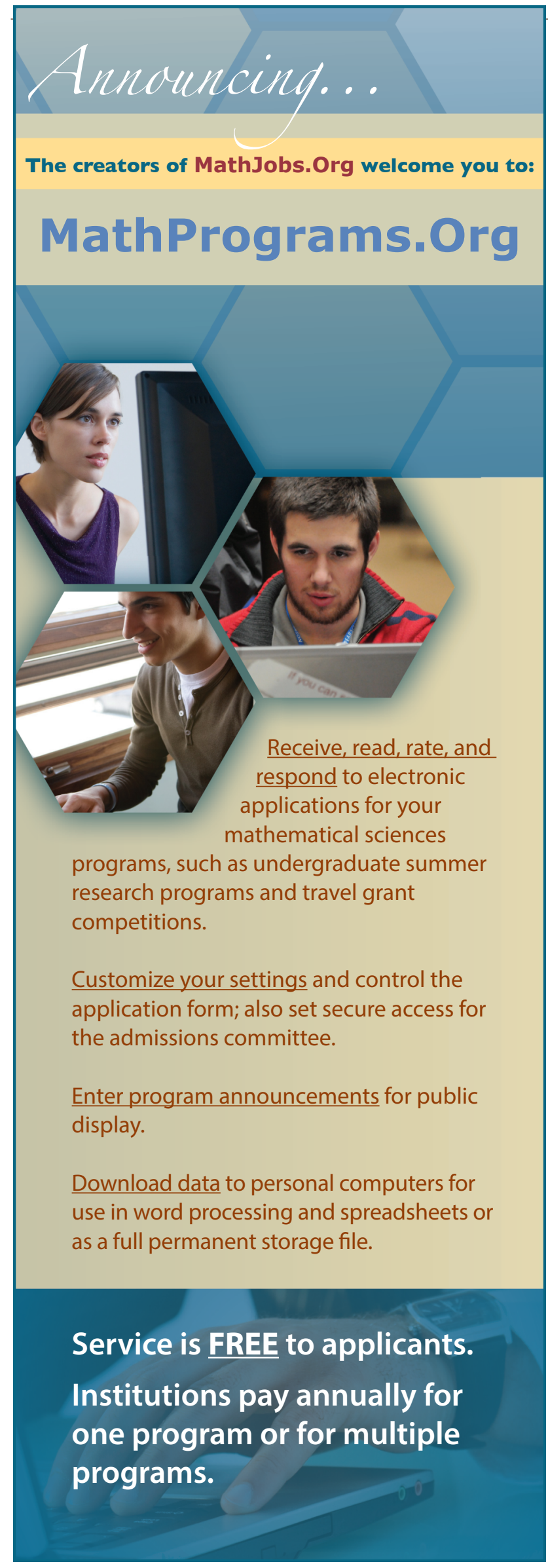

\section{Photo Credits}

Photo of June Huh is courtesy of Nayoung Kim. Photo of Eric Katz is courtesy of Joseph Rabinoff.

THE AUTHORS

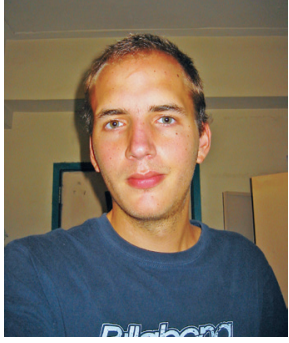

Karim Adiprasito

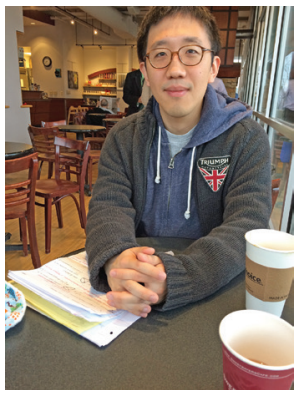

June Huh

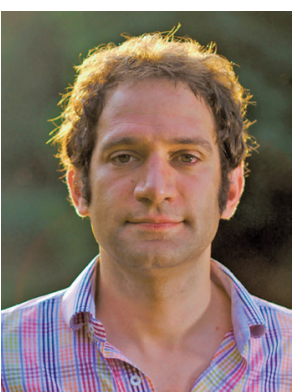

Eric Katz 